\title{
Prolyl hydroxylase 3 involvement in lung cancer progression under hypoxic conditions: association with hypoxia-inducible factor-1 $\alpha$ and pyruvate kinase M2
}

\author{
Xiao Chu ${ }^{1}$, Ming Xiang ${ }^{1}$, Liang Feng ${ }^{1}$, Hui Liu ${ }^{1}$, Chao Zhou $^{2}$ \\ ${ }^{1}$ Department of Thoracic Surgery, Fifth People's Hospital of Shanghai Fudan University, Shanghai 200240, China; ${ }^{2}$ Department of Thoracic Surgery, \\ Shanghai Jiao Tong University Affiliated Chest Hospital, Shanghai 200030, China \\ Contributions: (I) Conception and design: X Chu, H Liu; (II) Administrative support: None; (III) Provision of study materials or patients: None; (IV) \\ Collection and assembly of data: X Chu, H Liu; (V) Data analysis and interpretation: X Chu, H Liu; (VI) Manuscript writing: All authors; (VII) Final \\ approval of manuscript: All authors. \\ Correspondence to: Hui Liu. Department of Thoracic Surgery, Fifth People's Hospital of Shanghai Fudan University, No. 801, Heqing Road, Minhang \\ District, Shanghai 200240, China. Email: liuhui@5thhospital.com; Chao Zhou. Department of Thoracic Surgery, Shanghai Chest Hospital, Shanghai \\ Jiao Tong University, Shanghai 200030, China. Email: jesus8@163.com.
}

\begin{abstract}
Background: Previous studies have suggested that the functions of prolyl hydroxylase 3 (PHD3) in tumor growth, apoptosis and angiogenesis are essentially dependent on hypoxia-inducible factor (HIF)- $1 \alpha$ signaling. Nevertheless, whether PHD3 represents a promising tumor suppressor target remains to be clarified. To provide insight into the therapeutic potential of PHD3 in lung cancer, this study examined the effects of PHD3 expression on HIF-1 $\alpha$ and pyruvate kinase M2 (PKM2), as well as on lung cancer cell proliferation, migration, and invasion.

Methods: The model of hypoxia was established in A549 and SK-MES-1 cells with $200 \mu$ CoCl2 treatment, and verified by western blot and immunocytochemical staining. The expression levels of PKM2 and HIF-1 $\alpha$ were determined by western blot after overexpression or depletion of PHD3 in A549 and SKMES-1 cells. In addition, cell viability, migration and invasion were measured, respectively.

Results: Establishment of hypoxia in A549 and SK-MES-1 cells resulted in significant decreases in PHD3 expression and remarkable increase in PKM2 expression in 24 hrs. Overexpression of PHD3 in A549 and SK-MES-1 cells decreased HIF-1 $\alpha$ and PKM2 expression. In contrast, PHD3 knockdown increased HIF$1 \alpha$ and PKM2 $(\mathrm{P}<0.05)$. In addition, the viability, migration and invasion of A549 and SK-MES-1 cells were significantly decreased with PHD3 overexpression, but dramatically increased with $\mathrm{PHD} 3$ depletion $(\mathrm{P}<0.05)$. Conclusions: PHD3 is involved in lung cancer progression, and might be a promising therapeutic target for cancers.
\end{abstract}

Keywords: Prolyl hydroxylase 3 (PHD3); lung cancer; hypoxia-inducible factor- $1 \alpha$ (HIF-1 $\alpha$ ); pyruvate kinase M2 (PKM2)

Submitted Sep 21, 2018. Accepted for publication Aug 06, 2019.

doi: $10.21037 /$ jtd.2019.08.124

View this article at: http://dx.doi.org/10.21037/jtd.2019.08.124

\section{Introduction}

Microenvironmental hypoxia, a classic feature of many human malignancies, plays an important role in the growth and progression of tumors, promoting angiogenesis, metastasis, and resistance to therapy (1). Hypoxia-inducible factors (HIFs) are master regulators of oxygen homeostasis in response to hypoxia. HIF-1, a heterodimeric complex protein is composed of a constitutively expressed nuclear subunit HIF-1 $\beta$ and an oxygen dependent subunit (HIF-1 $\alpha$ ) both of which are ubiquitously expressed in human tissues (2). HIF-1 mediates the transcription of genes involved in 
oxygen delivery and metabolic adaptation to hypoxia, and plays essential roles in tumor proliferation, metabolism, angiogenesis, metastasis and differentiation (3). The expression of HIF- $1 \alpha$ correlates with poor patient prognosis in a variety of cancers (4-6). Evidence demonstrates that down-regulation of HIF- $1 \alpha$ inhibits the proliferation and invasion of lung cancer cells (7). Currently, HIF-1 $\alpha$ inhibitors acting through several molecular mechanisms are being investigated for therapeutic purposes (8).

Three HIF prolyl hydroxylase (PHD1, PHD2 and PHD3) which are oxygen sensors regulate the stability of HIFs by post-translational hydroxylation of proline residues in its $\alpha$ subunit in an oxygen-dependent manner, promoting the ubiquitination of HIF- $\alpha$ (9). The prolyl hydroxylase 3 (PHD3), which is regulated by hypoxia, could play important roles in tumor progression by controlling epidermal growth factor receptor (EGFR) activity (10) and hypoxic vascular endothelial growth factor secretion within the hypoxic microenvironment of the tumor (11). Pyruvate kinase M2 (PKM2) has emerging roles in cancer metabolism, growth and progression (12). Miao et al. demonstrated that down-regulation of PKM2 could inhibit cell proliferation, suppress cell migration and invasion, and induce apoptosis (13). Luo et al. reported that PHD3 mediates the binding of PKM2 to HIF-1 $\alpha$, and regulates PKM2 function (14). In addition, HIF-1 activates the transcription of PKM2 and PHD3.

Previous studies have suggested that the functions of PHD3 in tumor growth, apoptosis and angiogenesis are essentially dependent on HIF-1 signaling (11). It is also reported that expression of PHDs and of the asparagine hydroxylase FIH relates to an activated HIF pathway in non-small cell lung cancer (15). Moreover, Tennant et al. found that PHD3 was required to induce apoptosis and inhibit tumor growth in vivo, indicating that PHD3 could be a valid target for anti-cancer therapies (16). Nevertheless, whether PHD3 represents a promising tumor suppressor target remains to be clarified. To provide insight into the therapeutic potential of PHD3 in cancers, this study examined the effects of PHD3 dysregulation on HIF-1 $\alpha$ and PKM2 expression, as well as on the lung cell proliferation, migration, and invasion.

\section{Methods}

\section{Cell culture and induction of bypoxia}

A non-small-cell lung cancer cell line A549 and a lung squamous cell carcinoma cell line SK-MES-1 were purchased from American Type Culture Collection (Manassas, VA, USA). They were cultured in DMEM (12430-054, Gibco, Carlsbad, CA, USA) with $10 \%$ fetal bovine serum (FBS), 100,000 U/L penicillin and $100 \mathrm{mg} / \mathrm{L}$ streptomycin (15140, Gibco) for $48 \mathrm{~h}$ at $37^{\circ} \mathrm{C}$ in a $5 \% \mathrm{CO}_{2}$ atmosphere. A549 $\left(2 \times 10^{5}\right.$ cells per well) and SK-MES- 1 $\left(2 \times 10^{6}\right.$ cells per well) cells were seeded onto six-well plates and incubated overnight. Cells were then treated with $200 \mu \mathrm{M}$ cobalt chloride $\left(\mathrm{CoCl}_{2}\right.$, Sinopharm Chemical, USA) for 12 or $24 \mathrm{~h}$.

\section{Detection of HIF-1a, PHD3, and PKM2 expression}

For detection of HIF-1 $\alpha$, PHD3, and PKM2 protein expression, western blots were performed as previously described by Acker et al. (17), hypoxia-treated A549 and SKMES-1 cells were lysed in RIPA buffer (Beyotime, Jiangsu, China), and lysate protein concentration was measured with a BCA Protein Assay Kit (P0010S, Beyotime). Western blots were conducted using the following antibodies: HIF$1 \alpha$ (1:1,000, 20960-1-AP, Proteintech), PKM2 (1:1,000, 15822-1-AP, Proteintech), and PHD3 (1:1,000, 18325-1-AP, Proteintech). GAPDH (10494-1-AP, Proteintech, 1:1,000) was used as a loading control. Secondary antibodies were conjugated to horseradish peroxidase $(1: 10,000$, Jackson ImmunoResearch, West Grove, PA, USA) and protein signals were detected by enhanced chemiluminescence.

Immunocytochemical staining was carried out as in Comati et al. (18) using the antibodies listed above. Antigenantibody complexes were visualized with diaminobenzidine solution (Beijing Zhongshan Jinqiao Biological Technology Co., Beijing, China) and nuclei were labeled with hematoxylin (517-28-2, Solarbio, China).

\section{Plasmids and cell transfection}

DNA encoding the human PHD3 gene was synthesized by Sangon Biotech (Shanghai, China), inserted into pUC57, and transformed into E. coli One Shot Top10 competent cells. The recombinant plasmids were extracted using standard methods and verified by sequencing. The target gene was then digested from the pUC57 vector and ligated into the pcDNA3.1 expression vector to construct overexpression vectors, named pcDNA3.1-PHD3.

A549 and SK-MES-1 cells grown in six-well plates were transfected with pcDNA3.1-PHD3 or small interfering RNA (siRNA) against PHD3 (PHD3 siRNA, 
$100 \mathrm{nM}$ ) (diluted with $250 \mu \mathrm{L}$ OPTI-MEM, Tuoran biotend biotechnology Co., Ltd, Shanghai, China) using Lipofectamine 2000 (Thermo Fisher Scientific Inc., Shanghai, China) for $48 \mathrm{~h}$. The expression of PHD3 in A549 and SK-MES-1 cells after overexpression and knockdown was verified by western blot.

\section{Cell viability analysis}

The A549 and SK-MES-1 cells were incubated with $10 \mu \mathrm{L}$ CCK-8 (CK04, Dojindo Corp., Japan) for $1 \mathrm{~h}$ at $37^{\circ} \mathrm{C}$. The optical density (OD) at $450 \mathrm{~nm}$ was measured using an Infinite M1000 PRO plate reader (TECAN, Morrisville, NC, USA).

\section{Transwell and Matrigel invasion assays}

The A549 and SK-MES-1 cells were seeded into the upper chamber of Transwell plates (Corning Incorporated, Corning, NY, USA), and $600 \mu \mathrm{L}$ DMEM containing $10 \%$ FBS was added to the lower chamber. After $24 \mathrm{~h}$ incubation, cells in the bottom chamber were fixed with $4 \%$ buffered neutral formalin for $10 \mathrm{~min}$, stained with $0.1 \%$ crystal violet, and examined by electron microscopy $(200 x)$. For the Matrigel invasion assay, the experiment was conducted in Matrigel Matrix-coated Transwell chambers (Corning).

\section{Statistical analysis}

All data were presented as mean \pm standard error (SEM). GraphPad Prism 5.0 (GraphPad Software, San Diego, CA, USA) was used for data analysis. $\mathrm{P}<0.05$ indicated significant difference.

\section{Results}

\section{Influence of hypoxia on PHD3 and PKM2 expression}

We established a hypoxia model in A549 and SK-MES-1 cells, and examined the expression of HIF- $1 \alpha$ by western blot. As shown in Figure 1, the expression level of HIF$1 \alpha$ was increased in both A549 and SK-MES- 1 cells in a time-dependent manner $(\mathrm{P}<0.001)$. We also examined the expression levels of PHD3 and PKM2 by western blot (Figure 1A) and immunocytochemical staining (Figure 1B). The expression of PHD3 in hypoxic A549 and SK-MES-1 cells decreased over time, while PKM2 expression increased $(\mathrm{P}<0.01)$.

\section{Overexpression or knockdown of PHD3}

Since PHD3 expression decreased in hypoxic A549 and SK-MES-1 cells, we further investigated its role using overexpression and depletion experiments. As shown in Figure 2A, PHD3 expression was significantly increased in pcDNA3.1-PHD3-transfected A549 or SK-MES-1 cells compared to pcDNA3.1 control-transfected cells $(\mathrm{P}<0.001)$.

Three siRNA sequences were used to silence PHD3 expression. As shown in Figure 2B, PHD3 protein expression was significantly reduced in PHD3 siRNAtransfected A549 or SK-MES-1 cells compared to the controls $(\mathrm{P}<0.05)$. Among the three siRNAs, siRNA1 showed the highest silencing rate in both A549 and SKMES-1 cells and was used for further experiments.

\section{Effect of PHD3 expression changes on PKM2 and HIF-1a}

The effects of overexpression and knockdown of PHD3 on the expression of PKM2 and HIF1 $\alpha$ in A549 and SKMES- 1 cells were detected by western blot. The results showed that overexpression of PHD3 markedly decreased the expression of PKM2 and HIF-1 $\alpha$ in both A549 and SKMES-1 cells (Figure $3 A, \mathrm{P}<0.001$ ). Conversely, knockdown of PHD3 increased PKM2 and HIF-1 $\alpha$ expression in the two cell lines (Figure $3 B, \mathrm{P}<0.01$ ).

\section{Effect of PHD3 expression changes on lung cancer cells}

The effects of PHD3 overexpression and knockdown on cell viability, migration and invasion of A549 and SK-MES-1 cells under hypoxic conditions were assessed, respectively. As shown in Figure $4 A, B, C$, the viability, migration, and invasion were significantly decreased in A549 cells overexpressing PHD3 under hypoxic conditions, compared to negative controls $(\mathrm{P}<0.05)$. Conversely, the viability, migration, and invasion of A549 cells were significantly increased after PHD3 knockdown (Figure 4D, E, F, G, H, $\mathrm{P}<0.05)$. In addition, compared to negative controls, the viability, migration, and invasion of SK-MES-1 cells were also significantly decreased after overexpression of PHD3 under hypoxic conditions (Figure $5 A, B, C, D, E$, $\mathrm{P}<0.001)$, but remarkably increased after PHD3 knockdown (Figure $5 F, G, H, I, \mathcal{7}, \mathrm{P}<0.05$ ).

\section{Discussion}

Hypoxia is associated with prognosis and tumor control 


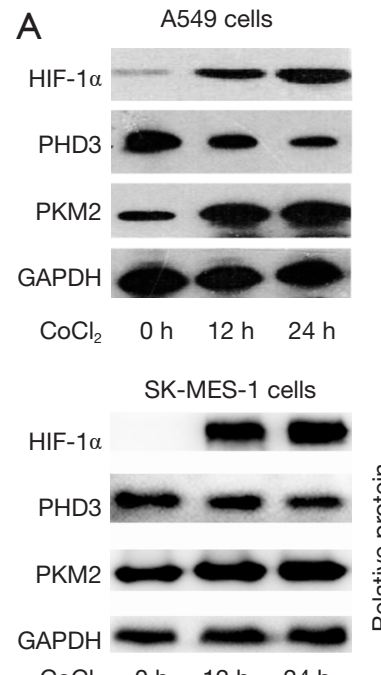

$\mathrm{CoCl}_{2} \quad \mathrm{Oh} \quad 12 \mathrm{~h} \quad 24 \mathrm{~h}$
A549 cells

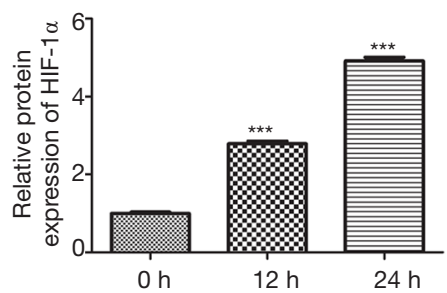

SK-MES-1 cells

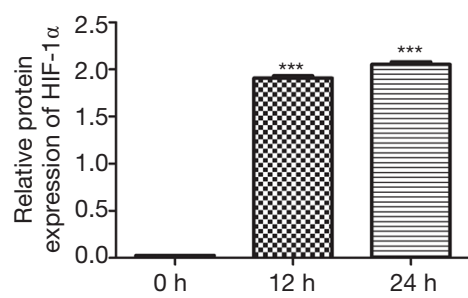

A549 cells

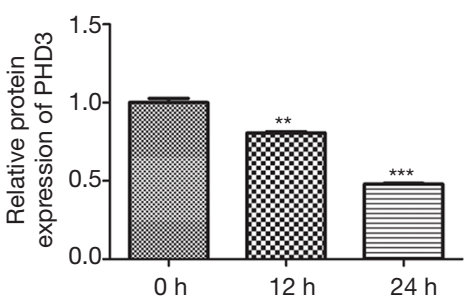

SK-MES-1 cells

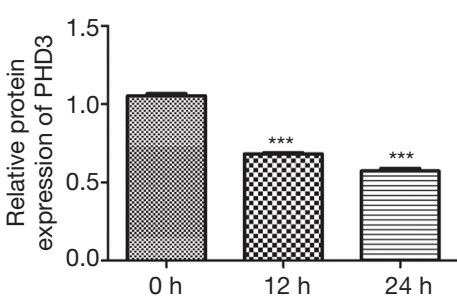

A549 cells

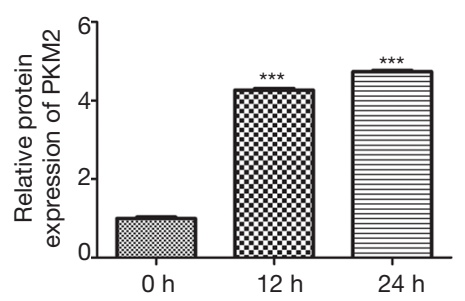

SK-MES-1 cells

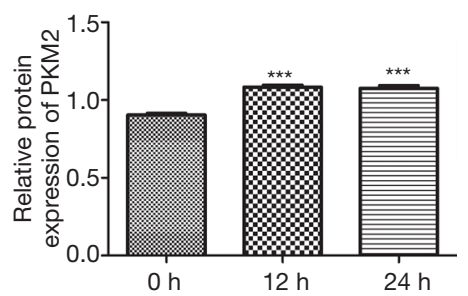

B
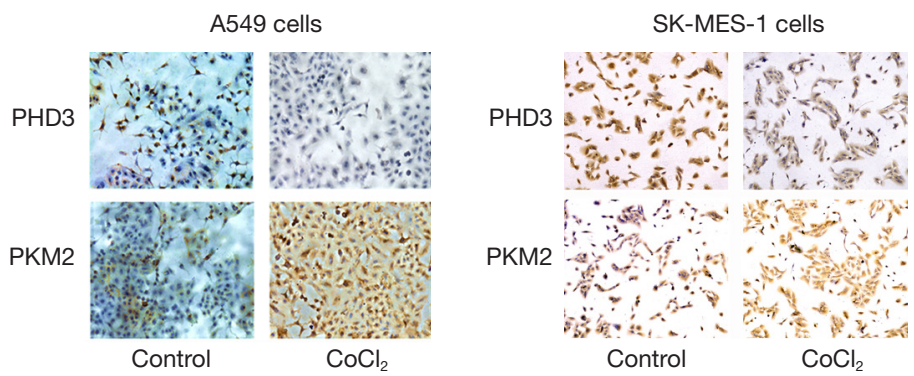

Figure 1 Expression of HIF-1 $\alpha$, PHD3, and PKM2 in hypoxic A549 and SK-MES-1 cells, detected by western blot (A) and immunohistochemistry (B). ${ }^{* *} \mathrm{P}<0.01$, and ${ }^{* * *} \mathrm{P}<0.001$. Magnification: $100 \times$.

A

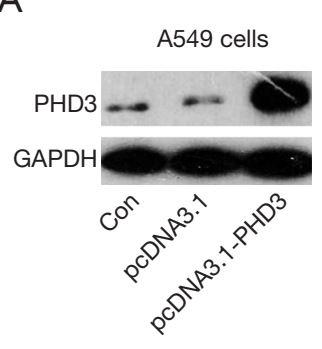

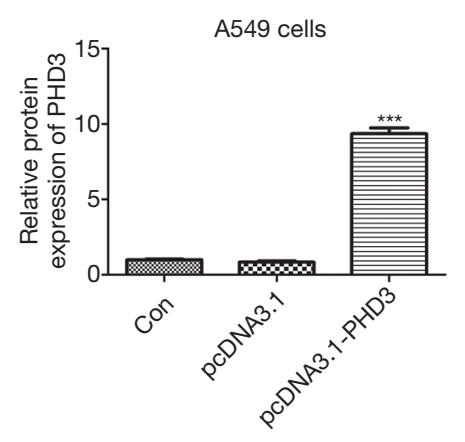

B

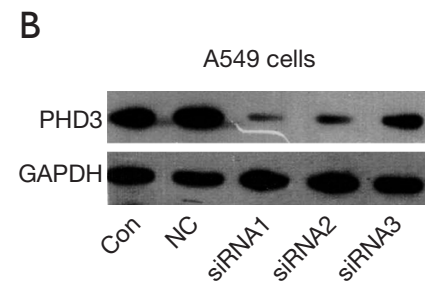

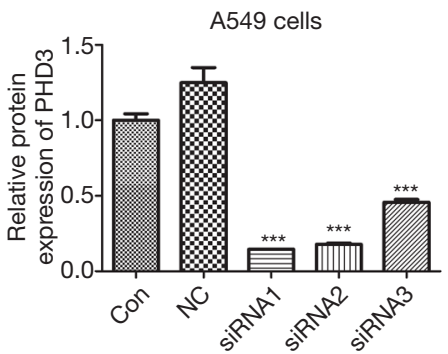
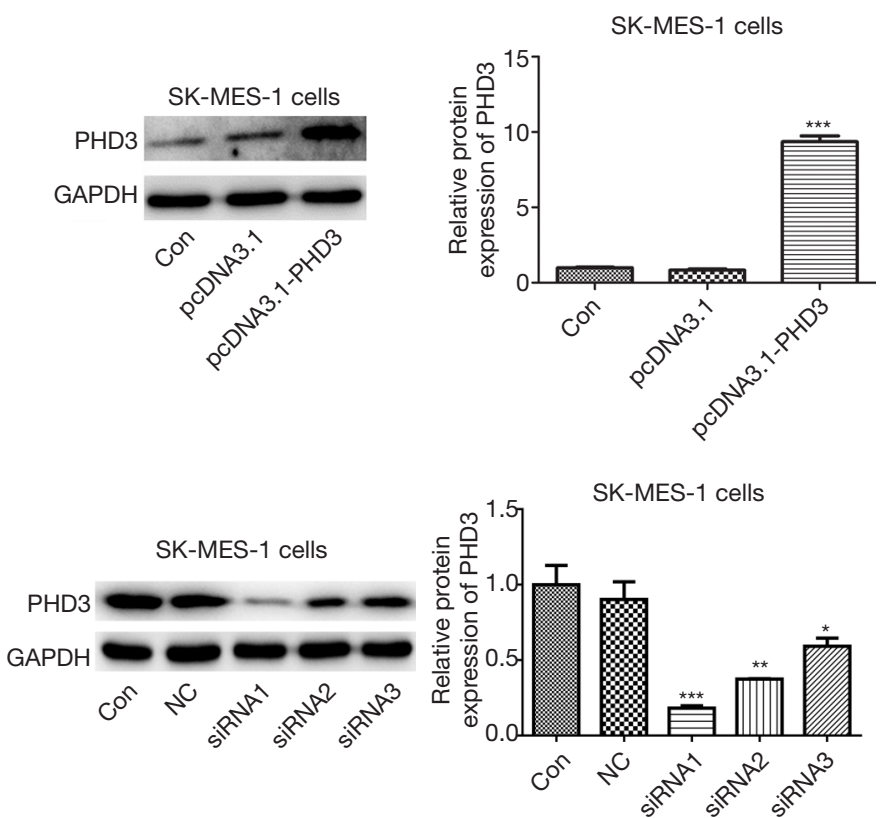

Figure 2 PHD3 protein levels in A549 and SK-MES-1 cells with overexpression (A) or knockdown (B) of PHD3. Con: control, NC: negative control. ${ }^{*} \mathrm{P}<0.05,{ }^{* *} \mathrm{P}<0.01$, and ${ }^{* * *} \mathrm{P}<0.001$. 
A
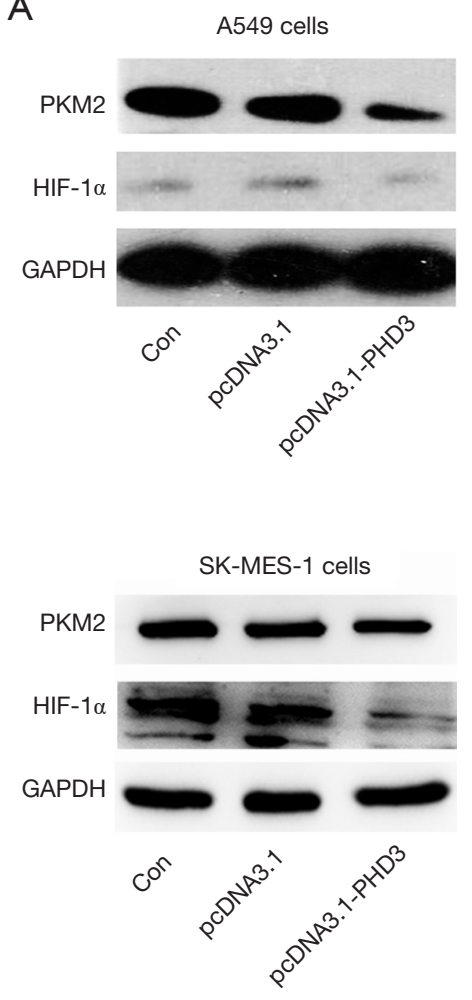

B
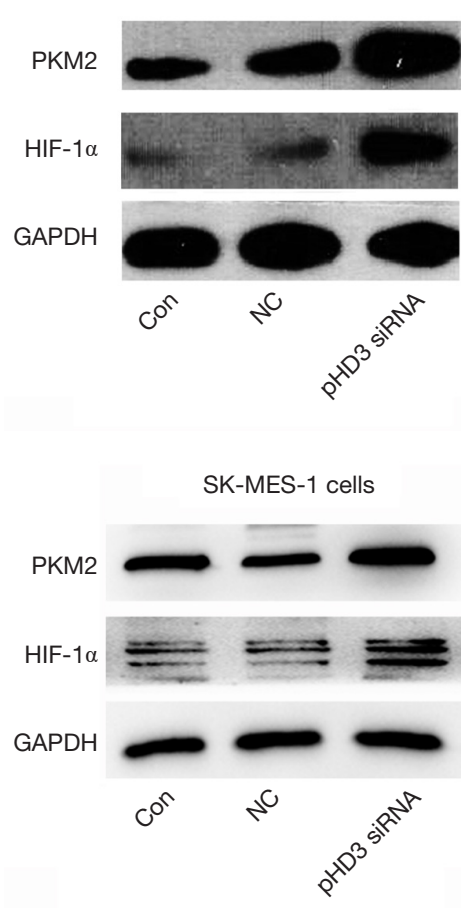
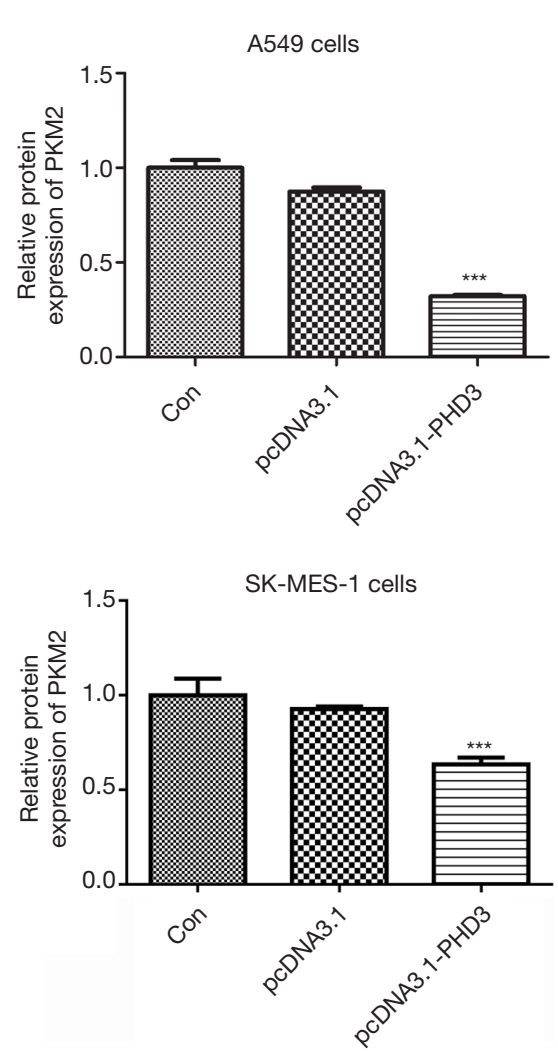

A549 cells
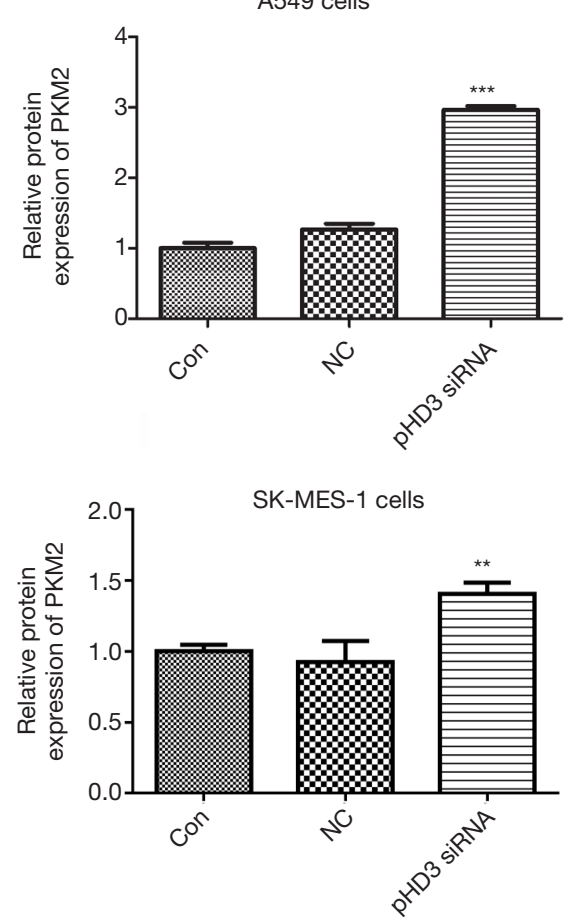
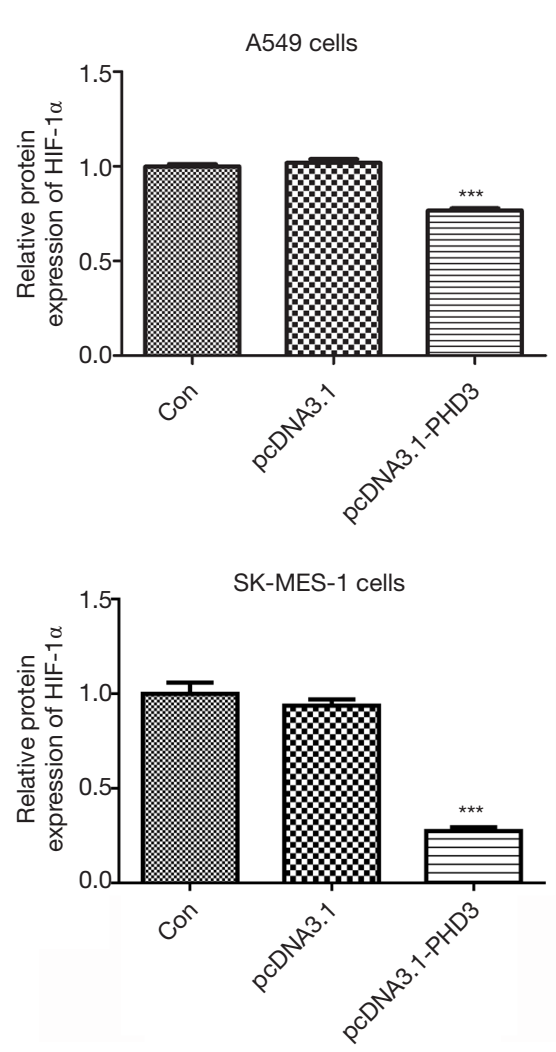

A549 cells
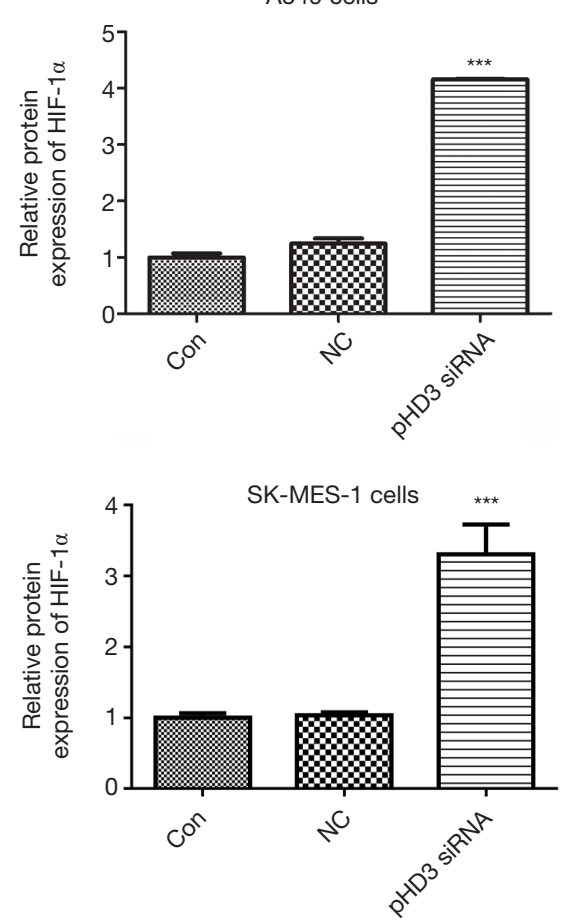

Figure 3 Expression of HIF-1 $\alpha$ and PKM2 in A549 and SK-MES-1 cells with overexpression (A) or knockdown (B) of PHD3. Con: control, NC: negative control. ${ }^{* *} \mathrm{P}<0.01$, and ${ }^{* * *} \mathrm{P}<0.001$. 

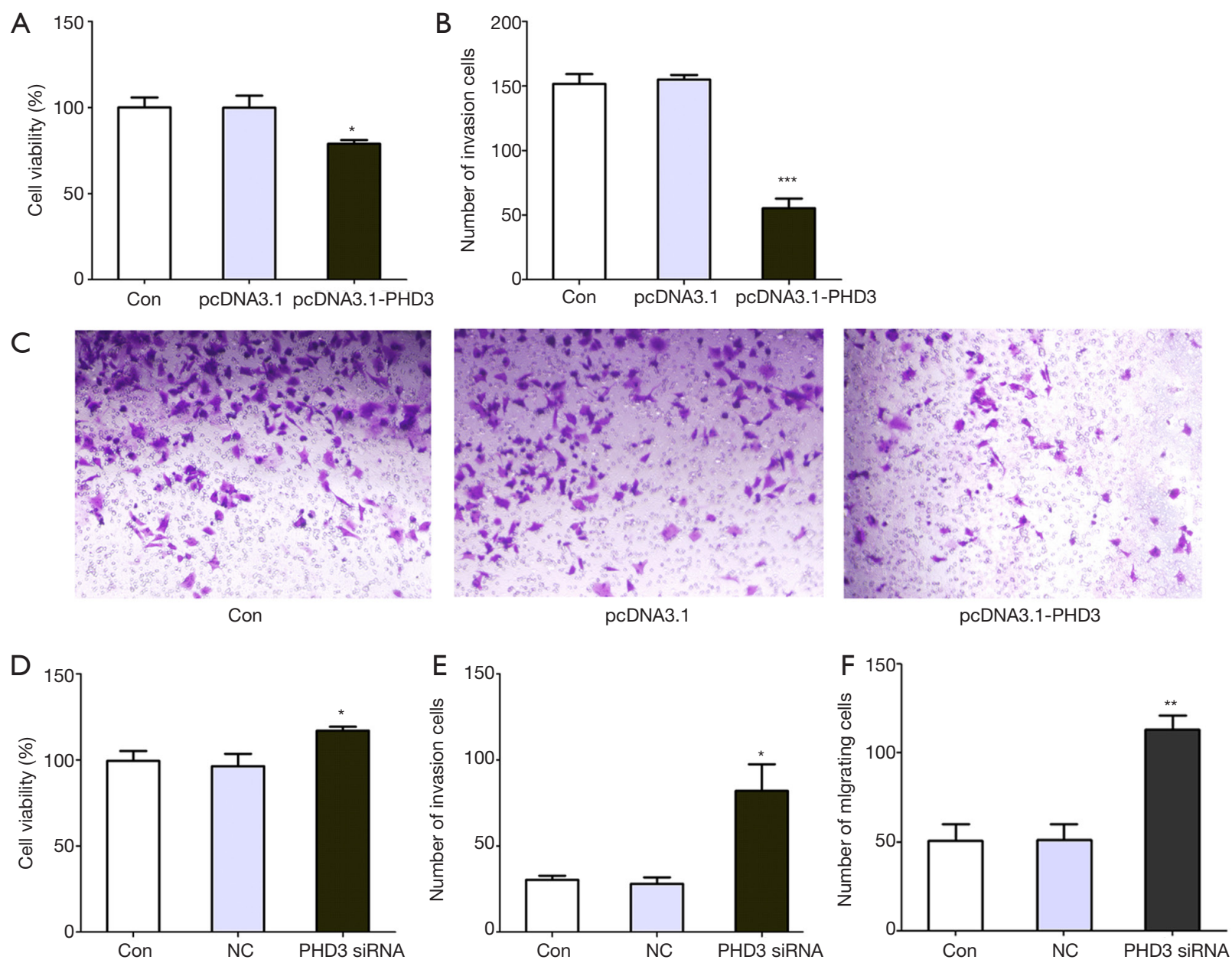

G

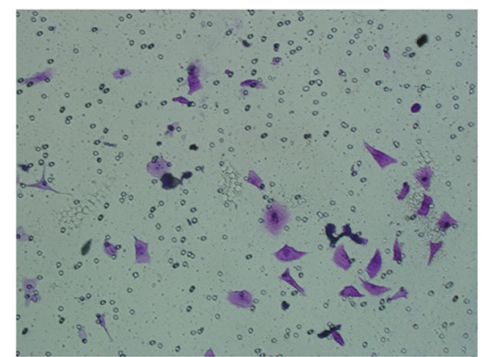

Con
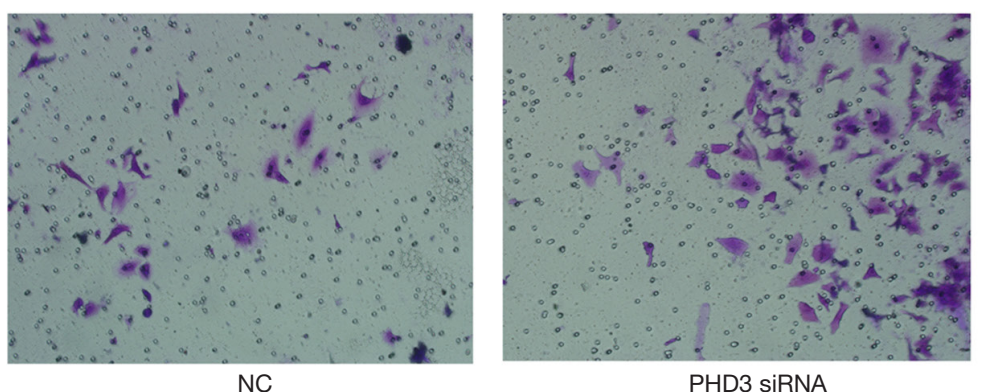

$\mathrm{H}$
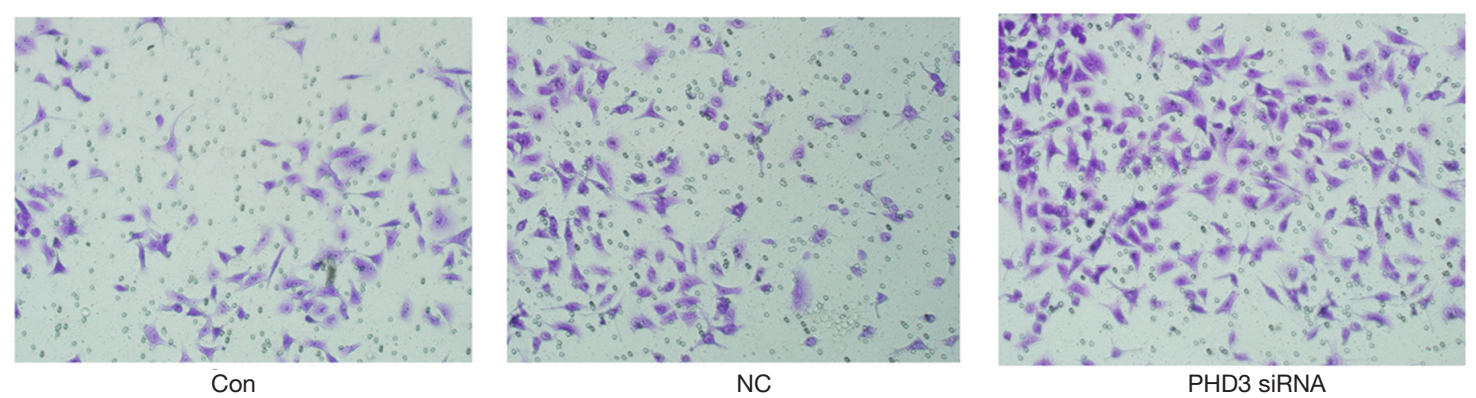

Figure 4 Cell viability (A) and invasion (B-C) were examined in PHD3-overexpressing A549 cells. Cell viability (D), invasion (E and G), and migration (F and $\mathrm{H}$ ) were examined in PHD3-depleted A549 cells. Con: control, NC: negative control. ${ }^{*} \mathrm{P}<0.05,{ }^{* *} \mathrm{P}<0.01$ and ${ }^{* * *} \mathrm{P}<0.001$. Magnification: $100 \times$. 

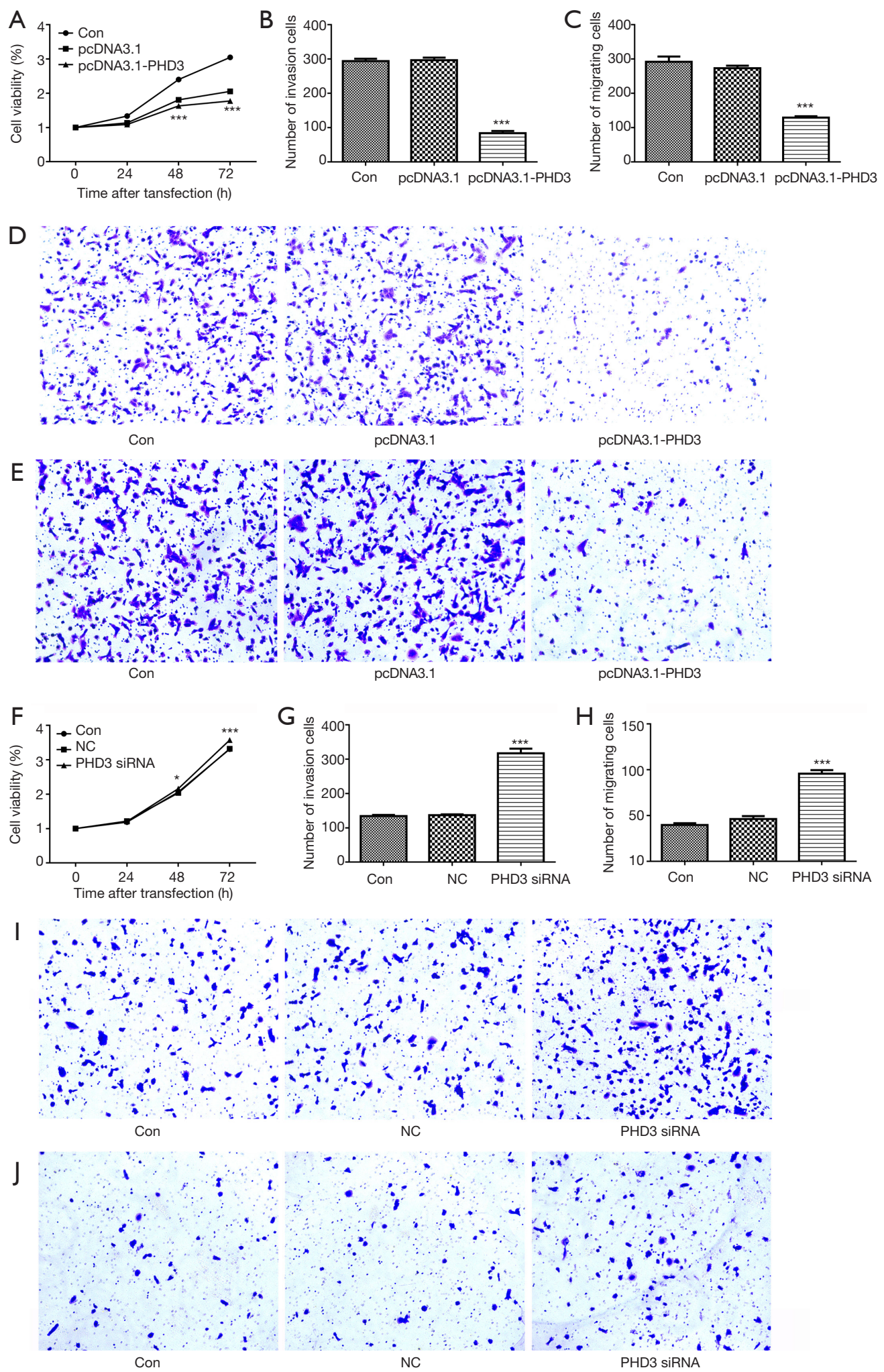

Figure 5 Cell viability (A), invasion (B and D), and migration (C and E) were examined in PHD3-overexpressing SK-MES-1 cells. Cell viability $(\mathrm{F})$, invasion ( $\mathrm{G}$ and $\mathrm{I})$, and migration $(\mathrm{H}$ and $\mathrm{J}$ ) were examined in PHD3-depleted SK-MES-1 cells. Con, control; NC, negative control. ${ }^{*} \mathrm{P}<0.05,{ }^{* * *} \mathrm{P}<0.001$. Magnification: $100 \times$. 
in many cancers, including lung cancer (19). Moreover, PHD3 has been reported to play crucial role of PHD3 in many types of cancers. For instance, PHD3 is shown to regulate differentiation, tumor growth and angiogenesis in pancreatic cancer (11); overexpression of PHD3 can inhibit the migratory and invasive capacity of gastric cancer cells (20); and PHD3 inhibits lung cancer metastasis and restored sensitivity to erlotinib (21). However, the regulatory mechanism of PHD3 in lung cancer has not been clarified. This study investigated the effects of PHD3 expression on the expression of PKM 2 and HIF-1 $\alpha$, as well as cell biological characteristics of lung cancer cells, to examine the mechanism of action and therapeutic potential of PHD3 in lung cancer.

$\mathrm{CoCl}_{2}$ is a hypoxia-mimicking agent, commonly used to induce hypoxia in vitro (22). After treatment with $\mathrm{CoCl}_{2}$, the expression of PKM2 and HIF-1 $\alpha$ was significantly increased in A549 and SK-MES-1 cells, while PHD3 expression was relatively decreased. This was consistent with previous studies (23-25), indicating that the hypoxic model was constructed successfully. PHDs, which can hydroxylate key proline residues in HIF- $1 \alpha$, are strikingly sensitive to graded levels of hypoxia in vitro (26). It is thought that $\mathrm{CoCl}_{2}$ stabilizes HIF-1 $\alpha$ via inhibiting the activity of PHD enzymes (27).

To further explore the relationship between PHD3, HIF$1 \alpha$, and PKM2, we examined the effects of overexpression and knockdown of PHD3. Increased expression of PHD3 is induced by HIF-1 under hypoxic conditions (14). Interaction of PKM2 with PHD3 enhances PKM2 binding to HIF-l $\alpha$ (14). PHDs inactivate HIFs in normoxia by activating degradation of the HIF- $\alpha$ subunit (28). In this study, PHD3 overexpression remarkably inhibited PKM2 and HIF- $1 \alpha$ expression, while knockdown of PHD3 significantly upregulated their expression, suggesting that expression of HIF- $1 \alpha$ and PKM2 were significantly negatively influenced by the expression level of PHD3. Thus, we hypothesize that a feedback loop involving HIF-1 $\alpha$, PKM2, and PHD3 could participate in cancer progression.

The dimer form of PKM2 leads to anabolic metabolism of glucose for energy production, and contributes to the synthesis of DNA and lipids required for cell proliferation and tumor growth (12). Chu et al. demonstrated that apoptosis and autophagy are significantly induced in A549 cells by inhibiting PKM2 expression (29). Sun et al. reported that PKM2 could regulate cell proliferation and invasion by activating glycolysis and lipid synthesis in lung adenocarcinoma (30), while Miao et al. reported that PKM2 could promote cell invasion in pancreatic ductal adenocarcinoma (13). PKM2 is also reported to control apoptosis in human leukemia cell lines (31). HIF-1 $\alpha$ accumulates due to decreased prolyl hydroxylase activity in hypoxia, potently enhances glycolytic metabolism, and interacts with c-Myc to control the cell cycle through the regulation of cyclins and cyclin-dependent kinase inhibitors (32). HIF- $1 \alpha$ also regulates migration in microglial cells (33). Additionally, it has been demonstrated that inhibition of HIF-1 $\alpha$ expression decreases proliferation and invasion of non-small cell lung cancer NCI-H157 cells (7) and enhances apoptosis (34). Increased PHD3 expression has been found to inhibit proliferation and enhance apoptosis through multiple mechanisms, including the regulation of EGFR (35) and HIF-1 signaling (36). Chen et al. demonstrated that enhanced PHD3 expression was negatively correlated with $\mathrm{Bcl}-2$ expression, thus playing potential role in inducing apoptosis in non-small cell lung cancer (37). In line with the previous studies abovementioned, we found that PHD3 overexpression significantly decreased the viability and invasiveness of lung cancer cells with the inhibition of HIF-1 $\alpha$ and PKM2 expression.

However, this study had also several limitations. First, HIF-1 $\alpha$ target gene expression was not detected to further state the regulation of PHD3 on HIF-1 $\alpha$-mediated biological processes. Second, $\mathrm{CoCl}_{2}$ treatment was used to mimic hypoxic conditions. Future studies are needed to determine the results in the actual hypoxic condition.

\section{Conclusions}

Taken together, this work suggests that PHD3, PKM2, and HIF-1 $\alpha$ may have linked roles in the control of proliferation, migration and invasion of lung cancer cells. In this study, abnormal expression of PHD3 significantly influenced lung cancer cells; however, the precise mechanism remains to be elucidated. Additional investigations are still required to investigate the roles of $\mathrm{PDH} 3$ in tumor development and its potential as a target for anti-cancer therapies.

\section{Acknowledgments}

Funding: This work was supported by the Natural Science Foundation of Minhang District of Shanghai (Project number: 2015MHZ032), the Young Scholars Scientific Research Project funded by The Fifth People's 
Hospital of Shanghai, Fudan University (Project number: 2015WYQJ01) and Scientific Research Project funded by The Fifth People's Hospital of Shanghai, Fudan University(Project number: 2019WYZT05).

\section{Footnote}

Conflicts of Interest: The authors have no conflicts of interest to declare.

Ethical Statement: The authors are accountable for all aspects of the work in ensuring that questions related to the accuracy or integrity of any part of the work are appropriately investigated and resolved. Ethical approval was obtained from the Fifth People's Hospital of Shanghai Fudan University Research Ethics Committee. Written informed consent was obtained for each participant, and the study was approved by the Hospital Ethics Committee.

\section{References}

1. Ikeda Y, Hisano H, Nishikawa Y, et al. Targeting and Treatment of Tumor Hypoxia by Newly Designed Prodrug Possessing High Permeability in Solid Tumors. Mol Pharm 2016;13:2283-9.

2. Fábián Z, Taylor CT, Lan KN. Understanding complexity in the HIF signaling pathway using systems biology and mathematical modeling. J Mol Med (Berl) 2016;94:377-90.

3. Weidemann A, Johnson RS. Biology of HIF-1 $\alpha$. Cell Death Differ 2008;15:621-7.

4. Baba Y, Nosho K, Shima K, et al. HIF1A Overexpression Is Associated with Poor Prognosis in a Cohort of 731 Colorectal Cancers. Am J Pathol 2010;176:2292-301.

5. Estevez-Garcia P, Lopezcalderero I, Molinapinelo S, et al. Spinophilin loss correlates with poor patient prognosis in advanced stages of colon carcinoma. Clin Cancer Res 2013;19:3925.

6. Choi H, Gillespie DL, Berg S, et al. Intermittent Induction of HIF-1 $\alpha$ Produces Lasting Effects on Malignant Progression Independent of Its Continued Expression. PLoS One 2015;10:e0125125.

7. Qian J, Bai H, Gao Z, et al. Downregulation of HIF$1 \alpha$ inhibits the proliferation and invasion of nonsmall cell lung cancer NCI-H157 cells. Oncol Lett 2016;11:1738-44.

8. Harada H. Hypoxia-inducible factor 1-mediated characteristic features of cancer cells for tumor radioresistance. J Radiat Res 2016;57:i99-i105.
9. Brocato J, Chervona Y, Costa M. Molecular responses to hypoxia-inducible factor $1 \alpha$ and beyond. Mol Pharmacol 2014;85:651.

10. Garvalov BK. PHD3 regulates EGFR internalization and signalling in tumours. Nat Commun 2014;5:5577.

11. Su Y, Loos M, Giese N, et al. PHD3 regulates differentiation, tumour growth and angiogenesis in pancreatic cancer. Br J Cancer 2010;103:1571-9.

12. Luo W, Semenza GL. Emerging roles of PKM2 in cell metabolism and cancer progression. Trends Endocrinol Metab 2012;23:560-6.

13. Miao Y, Lu M, Yan Q, et al. Inhibition of Proliferation, Migration, and Invasion by Knockdown of Pyruvate Kinase-M2 (PKM2) in Ovarian Cancer SKOV3 and OVCAR3 Cells. Oncol Res 2016;24:463-75.

14. Luo W, Hu H, Chang R, et al. Pyruvate kinase M2 is a PHD3-stimulated coactivator for hypoxia-inducible factor 1. Cell 2011;145:732.

15. Giatromanolaki A, Koukourakis MI, Pezzella F, et al. Expression of prolyl-hydroxylases PHD-1, 2 and 3 and of the asparagine hydroxylase FIH in non-small cell lung cancer relates to an activated HIF pathway. Cancer Lett 2008;262:87-93.

16. Tennant DA, Gottlieb E. HIF prolyl hydroxylase-3 mediates alpha-ketoglutarate-induced apoptosis and tumor suppression. J Mol Med (Berl) 2010;88:839-49.

17. Acker T, Diezjuan A, Aragones J, et al. Genetic evidence for a tumor suppressor role of HIF-2alpha. Cancer Cell 2005;8:131-41.

18. Comati A, Beck H, Halliday W, et al. Upregulation of Hypoxia-Inducible Factor (HIF)- $1 \alpha$ and HIF-2 $\alpha$ in Leptomeningeal Vascular Malformations of Sturge-Weber Syndrome. J Neuropathol Exp Neurol 2007;66:86-97.

19. Dehdashti F, Mintun MA, Lewis JS, et al. In vivo assessment of tumor hypoxia in lung cancer with $60 \mathrm{Cu}-$ ATSM. Eur J Nucl Med Mol Imaging 2003;30:844-50.

20. Xia YJ, Jiang XT, Jiang SB, et al. PHD3 affects gastric cancer progression by negatively regulating HIF1A. Mol Med Rep 2017;16:6882-9.

21. Dopeso H, Jiao HK, Cuesta AM, et al. PHD3 controls lung cancer metastasis and resistance to EGFR inhibitors through TGFa. Cancer Res 2018;78:1805-19.

22. Piret JP, Mottet D, Raes M, et al. CoCl2, a chemical inducer of hypoxia-inducible factor-1, and hypoxia reduce apoptotic cell death in hepatoma cell line HepG2. Ann N Y Acad Sci 2002;973:443-7.

23. Papadaki C, Sfakianaki M, Lagoudaki E, et al. PKM2 as a biomarker for chemosensitivity to front-line platinum- 
based chemotherapy in patients with metastatic non-smallcell lung cancer. Br J Cancer 2014;111:1757-64.

24. Pan K, Zhang H, Zhang ZM. Prolyl Hydroxulase 3 Regulating Expression of Hypoxia-inducible Factor- $1 \alpha$ in Non- small Cell Lung Cancer and Its Clinical Significance. Chinese General Practice 2014.

25. Fischer C, Leithner K, Wohlkoenig C, et al. Panobinostat reduces hypoxia-induced cisplatin resistance of non-small cell lung carcinoma cells via HIF-1 $\alpha$ destabilization. Mol Cancer 2015;50:S203-4.

26. Epstein AC, Gleadle JM, Mcneill LA, et al. C. elegans EGL-9 and mammalian homologs define a family of dioxygenases that regulate HIF by prolyl hydroxylation. Cell 2001;107:43-54.

27. Ho VT, Bunn HF. Effects of transition metals on the expression of the erythropoietin gene: further evidence that the oxygen sensor is a heme protein. Biochem Biophys Res Commun 1996;223:175-80.

28. Jokilehto T, Jaakkola PM. The role of HIF prolyl hydroxylases in tumour growth. J Cell Mol Med 2010;14:758-70.

29. Chu B, Wang J, Wang Y, et al. Knockdown of PKM2 induces apoptosis and autophagy in human A549 alveolar adenocarcinoma cells. Mol Med Rep 2015;12:4358.

30. Sun H, Zhu A, Zhang L, et al. Knockdown of PKM2 Suppresses Tumor Growth and Invasion in Lung Adenocarcinoma. Int J Mol Sci 2015;16:24574-87.

Cite this article as: Chu X, Xiang M, Feng L, Liu H, Zhou C. Prolyl hydroxylase 3 involvement in lung cancer progression under hypoxic conditions: association with hypoxia-inducible factor- $1 \alpha$ and pyruvate kinase M2. J Thorac Dis 2019;11(9):39413950. doi: $10.21037 /$ jtd.2019.08.124
31. Luo N, Zhao LC, Shi QQ, et al. Induction of Apoptosis in Human Leukemic Cell Lines by Diallyl Disulfide via Modulation of EGFR/ERK/PKM2 Signaling Pathways. Asian Pac J Cancer Prev 2015;16:3509-15.

32. Gordan JD, Thompson CB, Simon MC. HIF and c-Myc: Sibling Rivals for Control of Cancer Cell Metabolism and Proliferation. Cancer Cell 2007;12:108.

33. Qiao H, He X, Zhang Q, et al. A-synuclein induces microglial cell migration through stimulating HIF-1 $\alpha$ accumulation. J Neurosci Res 2017;95:1809-17.

34. Jiang Y, Wu GH, He GD, et al. The Effect of Silencing HIF-1 $\alpha$ Gene in BxPC-3 Cell Line on Glycolysis-Related Gene Expression, Cell Growth, Invasion, and Apoptosis. Nutr Cancer 2015;67:1314.

35. Henze AT, Garvalov BK, Seidel S, et al. Loss of PHD3 allows tumours to overcome hypoxic growth inhibition and sustain proliferation through EGFR. Nat Commun 2014;5:5582.

36. Tanaka T, Li TS, Urata Y, et al. Increased expression of PHD3 represses the HIF-1 signaling pathway and contributes to poor neovascularization in pancreatic ductal adenocarcinoma. J Gastroenterol 2015;50:975.

37. Chen S, Zhang J, Li X, et al. The expression of prolyl hydroxylase domain enzymes are up-regulated and negatively correlated with Bcl-2 in non-small cell lung cancer. Mol Cell Biochem 2011;358:257. 